\section{NSAIDs können erektile Dysfunktion begünstigen}

Männer, die regelmäßig nicht steroidale Antiphlogistika einnehmen, entwickeln einer aktuellen Studie zufolge 2,4 mal häufiger eine erektile Dysfunktion als Männer, die das nicht tun. Ein Grund, Alarm zu schlagen?

E igentlich könnte man annehmen, dass nicht steroidale Antiphlogistika (NSAIDs) einen positiven Effekt auf die Entstehung einer erektilen Dysfunktion (ED) ausüben. NSAIDs werden bei kardiovaskulären Erkrankungen eingesetzt, die wiederum mit ED in Verbindung gebracht werden. Bessern sich diese, sollte sich auch die ED bessern. In einer großen Kohortenstudie mit 80.966 Männern zwischen 45 und 69 Jahren haben US-amerikanische Wissenschaftler nun das Gegenteil herausgefunden.

Demnach ist die kontinuierliche Anwendung von NSAIDs mit einem signifikant erhöhten Risiko assoziiert, eine ED zu entwickeln $(\mathrm{OR}=2,40$; $95 \%$ CI 2,272,53). Nach Berücksichtigung von Faktoren wie Alter, Raucherstatus, Blutdruck, Diabetes mellitus, koronare Herzkrank und Body-Mass-Index blieb die Korrelation erhalten $(\mathrm{OR} 1,38)$. Insgesamt hatten von jenen Männer, die über einen Zeitraum von drei Monaten drei mal täglich NSAIDs einnahmen, 35,2\% eine ED, im Vergleich zu $24 \%$ bei Männern, die keine NSAIDs einnahmen.
Fazit: Männer, die regelmäßig NSAIDs einnehmen, bekommen häufiger eine ED als Männer, die das nicht tun. Zwar ist die Korrelation eindeutig, die Forscher können sie jedoch genauso wenig beweisen NSAIDs eine erektile Dysfunktion. wie alternative Erklärungen ausschließen. Sie betonen daher, dass Patienten nicht aufhören sollten, die verschriebenen NSAIDs einzunehmen. Stattdessen hoffen sie, Männer auf eine potenzielle Korrelation aufmerksam zu machen und grundsätzliche Risikofaktoren von ED ins Bewusstsein zu rücken.

slx

Gleason JM et al. Regular Nonsteroidal Anti-Inflammatory Drug Use and Erectile Dysfunction. J Urol 2011; 185: 1388-93

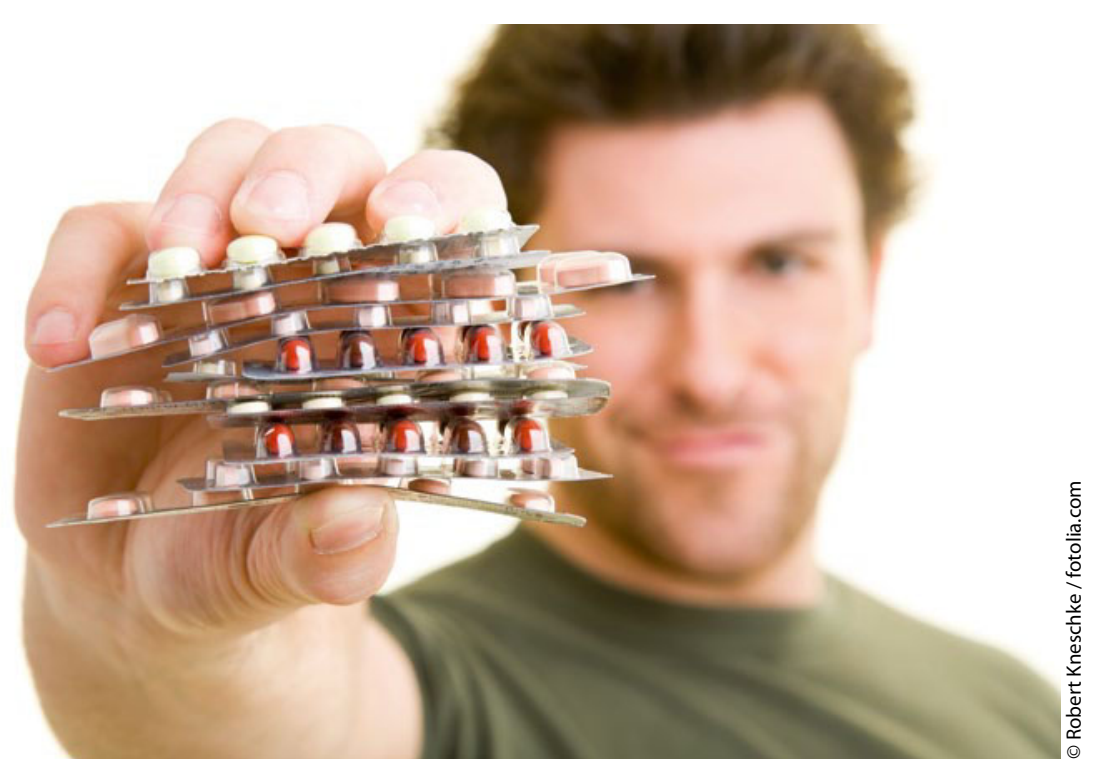

Bedenkenlos einnehmen oder nicht? Einer aktuellen Studie zufolge begünstigen

\section{Was ist teurer: radikale Prostatektomie oder Watchful-Waiting?}

Es gibt zahlreiche Studien, in denen das Gesamtüberleben und die Lebensqualität nach einer radikalen Prostatektomie im Vergleich zum Watchful-Waiting-Ansatz überprüft werden. Nun wurden erstmals die wirtschaftlichen Auswirkungen der beiden Strategien in einer randomisierten Studie untersucht.

D ie Watchful-Waiting-Strategie (WW) ist besonders für ältere Prostatakrebs-Patienten mit geringer Lebenserwartung interessant. So kann ihnen die Belastung einer radikalen Prostatektomie (RP) erspart werden. Die 2008 veröffentlichte Scandinavian Prostatic Cancer Group Study Number 4 (SPCG-4) ergab, dass RP-Patienten zwölf Jahre nach dem Eingriff einen Überlebensvorteil von 5,4\% gegenüber WW-Patienten hatten. Anhand von Patienten derselben Studienpopulation sollte nun herausgefunden werden, welcher der beiden Ansätze langfristig kostengünstiger ist. Die Forscher stellten die Hypothese auf, dass zunächst die RP teurer sein würde, im Laufe der Studiendauer WW aber durch häufigere Folgeuntersuchungen und Tumorprogression aufholen würde.

Von 212 randomisierten Patienten wurden 107 prostatektomiert, bei 105 wurde der WW-Ansatz verfolgt. Über einen Zeitraum von durchschnittlich zwölf Jahren wurden alle kostenverursachenden Posten wie Operation, Medikation, Krankenhausaufenthalte oder Laboruntersuchungen aufgezeichnet und anhand einer fixen Preisliste für jeden Patienten errechnet.

Die durchschnittlichen Gesamtkosten pro Patient waren in der RP-Gruppe um $34 \%$ höher als in der WW-Gruppe (24.247 EUR vs. 18.124 EUR; $\mathrm{p}<0,01$ ). Einzig die RP selbst war für diese Mehrkosten verantwortlich, andere Kostenpunkte wie medikamentöse Behandlung oder Untersuchungen unterschieden sich nicht signifikant zwischen den beiden Gruppen.

Die Autoren weisen jedoch darauf hin, dass ihre Studie vor dem „PSA-Zeitalter“ 
gestartet wurde und Patienten zum Zeitpunkt der Diagnose häufiger fortgeschrittene Tumoren hatten als heutzutage. $\mathrm{Zu}$ dem haben verbesserte Operationstechniken zu einer kürzeren Operationsdauer und Krankenhausverweildauer geführt. Für neue Methoden wie die robotergestütze laparoskopische Prostatektomie liegen derzeit noch keine Daten über
Langzeitkosten vor. Die Autoren vermuten, dass diese die der klassichen RP noch übersteigen.

Fazit: Eine radikale Prostatektomie inklusive aller Nachbehandlungen verursacht etwa um $34 \%$ höhere Kosten als Watchful Waiting. Dieser Unterschied, der fast ausschließlich auf die Kosten des
Eingriffs zurückzuführen sind, lässt sich im Laufe des Follow-up nicht mehr aufholen.

slx

Andersson SO et al. Managing localized prostate cancer by radical prostatectomy or watchful waiting: Cost analysis of a randomized trial (SPCG-4). Scand J Urol Nephrol. 2011; 45: 177-83

\section{Nierenteilresektion versus radikale Nephrektomie}

Eine Nierenteilresektion ist eine sichere Methode, um lokale Nierenzellkarzinome zu behandeln, mit einer etwas höheren Komplikationsrate als bei einer radikalen Nephrektomie. In einer internationalen Multicenterstudie wurde untersucht, inwiefern sich das onkologische Outcome der beiden Methoden unterscheidet.

$\mathrm{D}$ ie Nierenteilresektion (PN) gilt bei Nierenzellkarzinomen (RCC) im Stadium T1a $(<4 \mathrm{~cm})$ und gesunder kontralateraler Niere als Standardtherapie. Die Empfehlung, organerhaltend zu operieren, basiert auf Studien, in denen die onkologischen Ergebnisse unter NTR ähnlich gut waren wie nach einer radikalen Nephrektomie (RN), bei gleichzeitigem Erhalt der Nierenfunktion. Keine der bisherigen Vergleichsstudien war jedoch randomisiert.

1992 wurde eine internationale Multicenterstudie mit dem Ziel gestartet, Morbidität und Tumorkontrolle unter den beiden Operationsmethoden zu vergleichen. 541 Patienten mit Tumorstadium T1-T2 N0 M0 aus 17 Ländern wurden entweder einer Nierenteilresektion ( $\mathrm{n}=$ 268) oder einer radikalen Nephrektomie $(\mathrm{n}=273)$ zugeteilt. 55 Patienten wechselten die Behandlung: 16 der RN-Patienten $(5,9 \%)$ wollten auf eigenen Wunsch eine $\mathrm{RN}$, bei 39 der RN-Patienten (14,6\%) musste aus pathologischen Gründen eine $\mathrm{RN}$ durchgeführt werden.

Beide Methoden sind sicher, unter PN war die Komplikationsrate etwas höher (z.B. perioperative Blutungen als häufigste
Komplikation $28,3 \%$ vs. $17,5 \%$ ). Insgesamt verstarben nur 12 Patienten (2,2\%) tumorbedingt ( 4 in der RN-Gruppe, 8 in der PNGruppe). Aufgrund der wenigen Todesfälle war das Sterberisiko zwischen den beiden Methoden nicht signifikant. Die häufigsten Todesursachen waren kardiovaskuläre Ereignisse (9,3\% in der PN-Gruppe,
7,3\% in der RN-Gruppe). Auch das 10-Jahres-Überleben unterschied sich nicht signifikant $(75,7 \%$ bei PN, $81,1 \%$ bei $R N)$.

Fazit: Mit beiden Methoden lassen sich hervorragende onkologische Ergebnisse erzielen. Um eine Übertherapie zu vermeiden, sollte wenn möglich auf die organerhaltende Technik zurückgegriffen werden, so die Autoren.

van Poppel $\mathrm{H}$ et al. A prospective, randomised EORTC intergroup phase 3 study comparing the oncologic outcome of elective nephron-sparing surgery and radical nephrectomy for low-stage renal cell carcinoma. Eur Urol 2011; 59:543-52

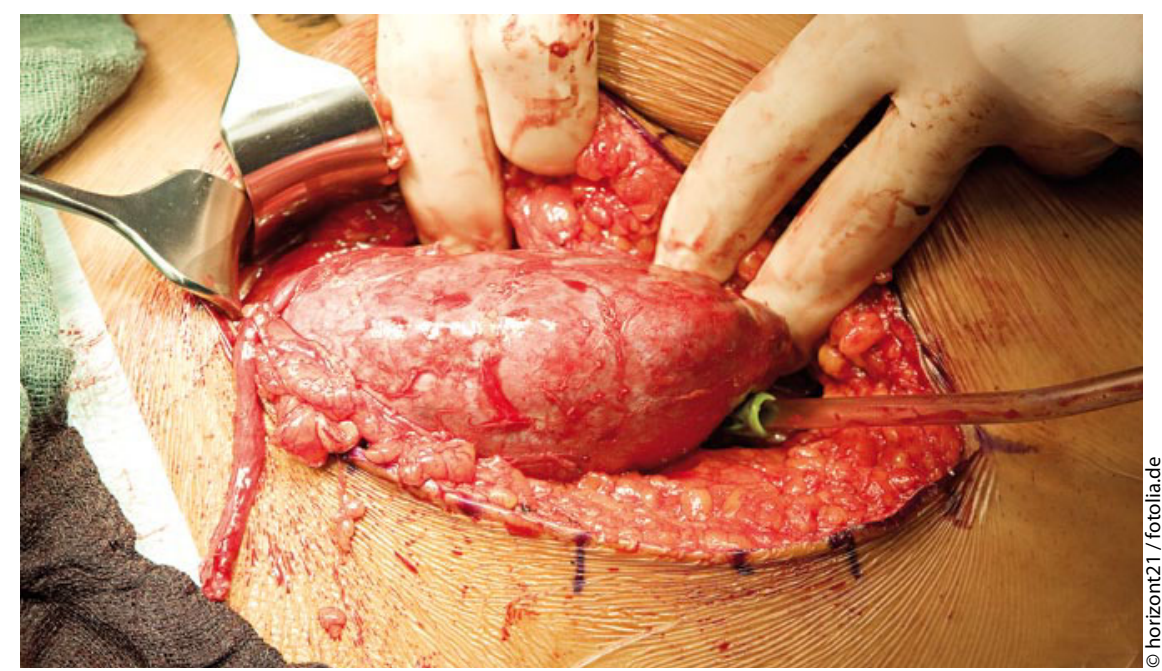

Nierenteilresektion und radikale Nephrektomie sind beides sichere Methoden zur Therapie eines Nierenzellkarzinoms. Doch wie unterscheidet sich das onkologische Outcome? 